\title{
LETTERS
}

\section{Systemic absorption of intranasal corticosteroids may occur and can potentially affect the hypothalamic- pituitary-adrenal axis}

In their recent CMAJ Practice article on intranasal corticosteroids, Drs. Fowler and Sowerby $^{1}$ stated that this therapy is not associated with hypothalamic-pituitary-adrenal (HPA) axis suppression. However, scenarios exist in which suppression may occur; therefore, some additional details may be useful. In support of their statement, the authors cited only a review article that, in turn, cited another review article and other small, older studies. Most studies looking at the association between intranasal corticosteroid use and HPA axis suppression have had major design flaws, namely small numbers; use of presently outdated and less accurate cortisol assays; collection of adrenal tests as an exploratory secondary outcome; lack of differentiation between different types, doses and durations of glucocorticoids; and lack of clinically relevant data.

A 2015 systematic review and metaanalysis of primary data, using appropriate inclusion, exclusion and stratification criteria showed that $4.2 \%$ (95\% confidence interval $0.5 \%-28.9 \%$ ) of patients using intranasal steroids could have adrenal suppression when a biochemical definition was used. ${ }^{2}$ The risk is likely increased with longer duration of use and with higher doses but is likely less than what is observed with inhaled steroids for asthma treatment.
However, in combination with medications that inhibit cytochrome P-450 3A4 (CYP3A4 inhibitors), which will slow systemic glucocorticoid metabolism, intranasal steroids may even cause Cushing syndrome, ${ }^{3,4}$ with rebound adrenal insufficiency after treatment is stopped, which emphasizes ${ }^{5}$ their potential for systemic absorption.

Although adverse clinical sequelae may be uncommon, modern laboratories' use of more specific cortisol immunoassays or mass spectrometry can show a low basal cortisol level when measured in a patient who is using intranasal or inhaled steroids, particularly in those receiving highly active antiretroviral therapy or azole antifungal agents. It is a common mistake to interpret this as "adrenal insufficiency" in need of replacement therapy instead of simply evidence that the synthetic glucocorticoid does indeed have systemic absorption and may be suppressing the HPA axis. A low level of serum cortisol in a patient using exogenous glucocorticoids is not an indicator of future HPA axis suppression, delayed recovery or clincial adrenal insufficiency after treatment is stopped. As intranasal steroids may be absorbed systemically and affect the HPA axis; decisions about when and how to stop such therapy should be carefully considered, in particular in patients undergoing treatment with CYP3A4 inhibitors.

\section{Gregory A. Kline MD}

Clinical professor, Medicine/

Endocrinology, University of Calgary, Calgary, Alta.

\section{Christopher J. Symonds MD}

Clinical associate professor, Medicine/ Endocrinology, University of Calgary,

Calgary, Alta.

\section{Daniel T. Holmes MD}

Clinical professor, Department of

Pathology and Lab Medicine, University of

British Columbia, Vancouver, BC

Cite as: CMAJ 2021 March 22;193:E426. doi: $10.1503 / \mathrm{cmaj} .78162$

\section{References}

1. Fowler J, Sowerby LJ. Using intranasal corticosteroids. CMAJ 2021;193:E47-47.

2. Broersen LHA, Pereira AM, Jørgensen JOL, et al. Adrenal insufficiency in corticosteroids use: systematic review and meta-analysis. J Clin Endocrinol Metab 2015;100:2171-80.

3. Hillebrand-Haverkort ME, Prummel MF, ten Veen $\mathrm{JH}$. Ritonavir-induced Cushing's syndrome in a patient treated with nasal fluticasone. AIDS 1999;13:1803-1803.

4. Chen F, Kearney T, Robinson S, et al. Cushing's syndrome and severe adrenal suppression in patients treated with ritonavir and inhaled nasal fluticasone. Sex Transm Infect 1999;75:274-274.

5. Veilleux O, Lee TC, McDonald EG. Rebound adrenal insufficiency after withdrawal of ritonavir in a 65-year-old man using inhaled budesonide. CMAJ 2017;189:E1188-91.

Competing interests: None declared.

Content licence: This is an Open Access article distributed in accordance with the terms of the Creative Commons Attribution (CC BY-NC-ND 4.0) licence, which permits use, distribution and reproduction in any medium, provided that the original publication is properly cited, the use is noncommercial (i.e., research or educational use), and no modifications or adaptations are made. See: https://creativecommons.org/ licenses/by-nc-nd/4.0/ 